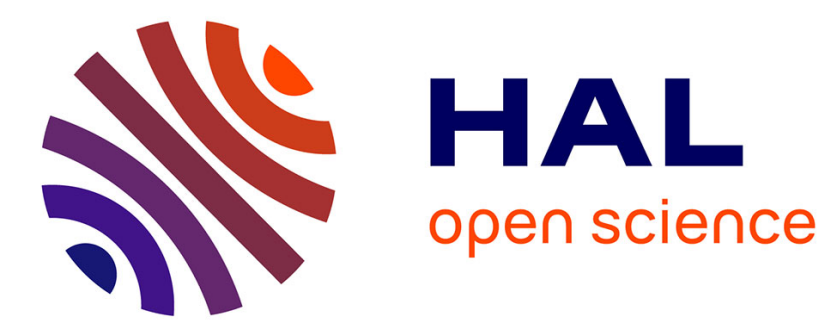

\title{
Photometry and colour index of Comet 67P/Churyumov-Gerasimenko on 2015 December 12
}

A. K. Sen, Edith Hadamcik, Robert Botet, Jérémie Lasue, S. Roy Choudhury, R. Gupta

\section{- To cite this version:}

A. K. Sen, Edith Hadamcik, Robert Botet, Jérémie Lasue, S. Roy Choudhury, et al.. Photometry and colour index of Comet 67P/Churyumov-Gerasimenko on 2015 December 12. Monthly Notices of the Royal Astronomical Society, 2019, 487 (4), pp.4809-4818. 10.1093/mnras/stz1538 . insu-02151608

\section{HAL Id: insu-02151608 https://hal-insu.archives-ouvertes.fr/insu-02151608}

Submitted on 11 Jun 2019

HAL is a multi-disciplinary open access archive for the deposit and dissemination of scientific research documents, whether they are published or not. The documents may come from teaching and research institutions in France or abroad, or from public or private research centers.
L'archive ouverte pluridisciplinaire $\mathbf{H A L}$, est destinée au dépôt et à la diffusion de documents scientifiques de niveau recherche, publiés ou non, émanant des établissements d'enseignement et de recherche français ou étrangers, des laboratoires publics ou privés. 


\title{
Photometry and Colour Index of Comet 67P/Churyumov-Gerasimenko on 12 December 2015
}

\author{
A. K. Sen,${ }^{1 \star}$ E. Hadamcik,${ }^{2} \dagger$ R. Botet,${ }^{3} \ddagger$ \\ J. Lasue ${ }^{4} \S$ S. Roy Choudhury, ${ }^{1}$ and R. Gupta ${ }^{5} \|$ \\ ${ }^{1}$ Department of Physics, Assam University, Silchar 788001, India \\ ${ }^{2}$ LATMOS/IPSL, UVSQ Université Paris-Saclay, Sorbonne Université , CNRS, Guyancourt, France \\ ${ }^{3}$ Laboratoire de Physique des Solides UMR8502, Université Paris-Sud,Université Paris-Saclay, Orsay 91405, France \\ ${ }^{4}$ IRAP, OMP, CNRS, Toulouse, France \\ ${ }^{5}$ IUCAA, Ganeshkhind, Pune 411007, India
}

Accepted XXX. Received YYY; in original form ZZZ

\begin{abstract}
Comet 67P/Churyumov-Gerasimenko (hereafter, 67P) was observed on 12 December 2015, from $2 \mathrm{~m}$ Himalayan Chandra Telescope in India in photometry to study its dust properties, using Bessell $\mathrm{R}$ and I filters. We study the photometric images to highlight coma structures and jets. The radial decrease in intensity in the different coma structures are compared to the azimuthally integrated intensities. The observations of the slopes show a quasi-steady-state coma to an optocentric distance of about $20000 \mathrm{~km}$. The change in the slopes in the structures indicates changing properties of the dust particles and/or change in their local size distributions. Comparison of the radial decrease in the two wavelengths suggests a change in the local colour-index. Based on the absolute photometry carried out in the present work, we calculate the reddening of the comet dust which helps to characterize variations in the size and the materials of the particles. The colour-index is calculated for different apertures and regions in the coma $(0.40 \pm 0.07 \mathrm{mag})$ for a 22000 diameter aperture. A colour map is constructed showing the variation of the colour-index through the coma. Changes appear at the transition between the coma and the tail with a low colour-index $(<0.3 \mathrm{mag})$ close to the optocentre and further away increasing up in the tail direction (about $0.45 \mathrm{mag}$ at $10000 \mathrm{~km}$ ). We interpret these changes in terms of dust properties and we compare our results to other remote observations of 67P including in situ Rosetta observations.
\end{abstract}

Key words: Comets general: Comets individual: 67P/Churyumov-Gerasimenko; Methods: observational; Techniques: photometric and polarimetric

\section{INTRODUCTION}

Comet 67P/Churyumov-Gerasimenko was discovered in 1969 (Churyumov 2005). The comet remained at a large perihelion distance $(\mathrm{q}=2.7 \mathrm{au})$, while the encounter with Jupiter in 1959 reduced it to its present value of $\mathrm{q}=1.29$ au (Maquet (2015)) and the period stabilized at 6.45 julian years. The sideral rotation period changed slightly between 2009 and 2014 perihelion, respectively from $\mathrm{P}_{1}=12.76129 \pm 0.00005 \mathrm{~h}$ to $\mathrm{P}_{2}=12.404 \pm 0.007 \mathrm{~h}$ (Mottola et al. 2014). Numerous observations of the comet

\footnotetext{
* E-mail:asokesen@yahoo.com

† E-mail:edith.hadamcik@latmos.ipsl.fr

$\ddagger$ E-mail:robert.botet@u-psud.fr

$\S$ E-mail:jlasue@irap.omp.eu

๑ E-mail:saumya.viv@gmail.com

|| E-mail:rag@iucaa.in
}

were published before the rendezvous mission with Rosetta in 2014, such as: Osip et al. (1992) and A'Hearn et al. (1995) for the depletion of $\mathrm{C}_{2}$ to $\mathrm{CN}$; photometry observations by Schleicher (2006) who evidenced a peak value at $450 \mathrm{~cm}$ of the A $f \rho$ parameter (defined by A'Hearn et al. (1984) as a proxy measuring the dust production rate), coma features and colour index; Hadamcik et al. (2010) for polarization maps.

The European Space Agency's Rosetta spacecraft, launched in 2004, escorted the comet from 2014 to 2016 at distances from the surface as low as $6 \mathrm{~km}$ and up to 1000 $\mathrm{km}$ to observe the tail when comet activity increased. The spacecraft finally entered into contact with the surface on 30 September 2016 (Taylor et al. 2017). The onboard dust experiments (OSIRIS, GIADA, COSIMA, MIDAS) provided the ground-truth for the understanding of cometary dust 
and at least of 67P ( Rotundi et al. 2015; Hilchenbach et al. 2016; Bentley et al. 2016; Mannel et al. 2016; Della Corte et al. 2016). It also helps to understand the variations of its properties with perihelion distance, epoch and season. The size of the comet nucleus was estimated using different techniques before direct measurements from the Rosetta images which showed the bilobate shape of the comet nucleus with a large lobe $4.1 \times 3.3 \times 1.8 \mathrm{~km}$ and a small lobe $2.6 \times 2.3 \times 1.8 \mathrm{~km}$ (Sierks et al. 2015). Several experiments (COSIMA on Rosetta, COSAC and PTOLEMY onboard Philae) measured the composition of the dust and, in particular, the very high amount of refractory organics (50\% in mass) present in the dust particles (Schulz et al. 2015; Fray et al. 2016).

The European Space Agency encouraged and accompanied worldwide campaigns of remote observations from major observatories using techniques of diverse kinds during all the Rosetta mission (Snodgrass et al. 2017). These observations provided large-scale morphological studies and helped to follow evolution of the activity and spectroscopy of the comet along the orbit, complementing the extensive data from Rosetta observations. A review on cometary dust can be found in Levasseur-Regourd et al. (2018) and references therein. It compares the new results obtained with Rosetta to other results of previous space missions and remote observations and gives a very general picture of the present knowledge on cometary dust after Rosetta.

The dust coma morphology showed two main jet structures extending to more than $40000 \mathrm{~km}$, at least from June 2015 to January 2016, and a few shorter jets not always detected. One of the main jets is sunward. The other one seems to originate from the same region, but it is curved towards the north (e.g. Hadamcik et al. 2016 for HST and HCT observations; Rosenbush et al. 2017; Knight et al. 2017). The remote observations during the encounter help to understand the behaviour of the dust at large cometary distances. The present work is a part of this campaign. We will use some of the recent publications for discussions, together with results from Rosetta (e.g. Hadamcik et al. 2016) and various publications on the colour of the dust such as Bertini et al. (2017) and Frattin et al. (2017), and also Rosenbush et al. (2017) with colour and polarization maps obtained at different dates after perihelion.

We present hereafter the observation conditions and data reduction in Section 2, the discussion in Section 3 and conclusion in Section 4.

\section{OBSERVATIONS AND DATA REDUCTION}

For the observations, we used the 2-m Himalayan Chandra Telescope $\left(32^{\circ} .7794 \mathrm{~N} ; 78^{\circ} .9642 \mathrm{E}, 4500 \mathrm{~m}\right)$ of the Indian Astronomical Observatory Hanle. The telescope was remotely operated from the Centre for Research and Education in Science and Technology 'CREST', Bangaluru India. The sky was generally photometric. Details of the sky conditions are given in Table 1 . The observations were carried out at the Cassegrain focus (f/9 beam), which has an imaging device HFOSC with $2048 \times 2048$ EEV CCD detector, $15 \mu \mathrm{m}$ pixel size. The plate-scale on CCD was $0.296 \mathrm{arcsec} / \mathrm{pixel}$ resulting in a 10.1 arcminutes $\times 10.1$ arcminutes field of view for the CCD imaging area. The
Table 1. Sky conditions during the observation of comet $67 \mathrm{P}$ at Himalayan Chandra Telescope, India, on 12 December 2015.

\begin{tabular}{lcccr}
\hline UT & $12: 52$ & $17: 17$ & $20: 15$ & $00: 17$ \\
\hline Temperature $\left({ }^{\circ} \mathrm{C}\right)$ & -8.0 & -10.4 & -12.0 & -13.2 \\
Humidity $(\%)$ & 20 & 23 & 24 & 28 \\
Seeing (arcsec) & 1.9 & 1.8 & 2.2 & 2.8 \\
Sky & clear & clear & clear & clear \\
\hline
\end{tabular}

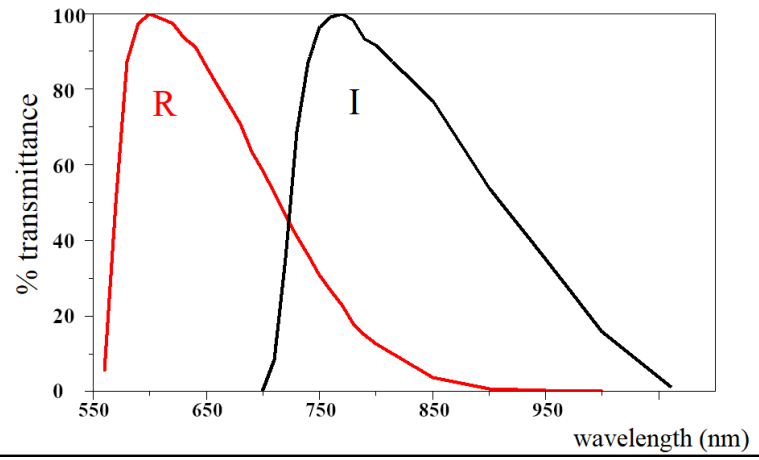

Figure 1. Normalised transmittances versus the wavelength in $\mathrm{nm}$ of the R (red) and I (infrared) Bessell filters used in the 12 December 2015 observations.

gain and read noise of the CCD were 1.22 electrons/Data Numbers and 4.8 electrons respectively.

We used Bessell red (centre wavelength: $\lambda=600 \mathrm{~nm}$, bandwith: $\Delta \lambda=140 \mathrm{~nm})$ and Bessell infrared $(\lambda=770 \mathrm{~nm}$, $\Delta \lambda=170 \mathrm{~nm}$ ) filters, the characteristics of which are given in Figure 1.

Fifteen bias frames were taken during the observation night. Moreover three evening twilight flats and three morning twilight flats were taken with both filters. The basic photometric reductions (bias-subtraction and flat fielding with specific data for near-infrared) were done to carry out absolute photometry, by observing two G-type photometric standard stars: SA 93424 and SA 96235.

The photometry was carried out taking the 'aperture photometry' within the IRAF task APPHOT. The observed magnitudes were corrected from atmospheric extinction at different airmass values using the extinction coefficients available from Parihar et al. (2003). The details of the observation log for these two photometric standard stars are available in Table 2 and for the comet $67 \mathrm{P}$ in Table 3 . The observed magnitudes of the two stars are shown in Table 4. We took 13 images of the comet, alternatively through the $\mathrm{R}$ and I filters, each with exposure time $600 \mathrm{~s}$ except for the image No1 (300 s). As indicated in the Table 3, some images were rejected due to trailing star image being superimposed on the comet region. We eventually accepted nine images for analysis, and two successive images with filter R and filter I (the images No 10 and 11) were used to build the colour index map under controlled sky conditions. 
Table 2. Observation log for two photometric standard stars SA $93424\left(2000 \mathrm{RA}=01^{\mathrm{h}} 55^{\mathrm{m}} 26^{\mathrm{s}}, \mathrm{DEC}=+00^{\circ} 56^{\prime} 54^{\prime \prime}\right)$ and $\mathrm{SA}$ $96235\left(2000 \mathrm{RA}=04^{\mathrm{h}} 53^{\mathrm{m}} 19^{\mathrm{s}}, \mathrm{DEC}=-00^{\circ} 04^{\prime} 42^{\prime \prime}\right)$ observed from Himalayan Chandra Telescope, India, on 12 December 2015.

\begin{tabular}{lcccr}
\hline $\begin{array}{l}\text { Serial } \\
\text { No }\end{array}$ & $\begin{array}{c}\text { Star } \\
\text { name }\end{array}$ & $\begin{array}{c}\text { UT } \\
\text { (HH:MM) }\end{array}$ & Filter & $\begin{array}{r}\text { Integration } \\
\text { Time (s) }\end{array}$ \\
\hline 1 & SA93424 & $13: 55$ & R & 3 \\
2 & SA93424 & $13: 52$ & I & 3 \\
3 & SA96235 & $19: 03$ & R & 5 \\
4 & SA96235 & $19: 05$ & I & 5 \\
\hline
\end{tabular}

Table 3. Observations of the comet $67 \mathrm{P}$ from Himalayan Chandra Telescope, India, on 12 December 2015. The tracking mode was non-sidereal/cometary. Four frames were rejected due to trailing star image being superimposed on comet image.

\begin{tabular}{lcccc}
\hline $\begin{array}{l}\text { Serial } \\
\text { No }\end{array}$ & $\begin{array}{c}\mathrm{UT} \\
(\mathrm{HH}: \mathrm{MM})\end{array}$ & Filter & $\begin{array}{c}\text { Integration } \\
\text { Time }(\mathrm{s})\end{array}$ & comment \\
\hline 1 & $21: 21$ & $\mathrm{R}$ & 300 & accepted \\
2 & $21: 29$ & $I$ & 600 & rejected \\
3 & $21: 42$ & $R$ & 600 & rejected \\
4 & $21: 55$ & $I$ & 600 & rejected \\
5 & $22: 07$ & $R$ & 600 & rejected \\
6 & $22: 19$ & $\mathrm{I}$ & 600 & accepted \\
7 & $22: 31$ & $\mathrm{R}$ & 600 & accepted \\
8 & $22: 43$ & $\mathrm{I}$ & 600 & accepted \\
9 & $22: 55$ & $\mathrm{R}$ & 600 & accepted \\
10 & $23: 07$ & $\mathrm{I}$ & 600 & accepted \\
11 & $23: 19$ & $\mathrm{R}$ & 600 & accepted \\
12 & $23: 35$ & $\mathrm{I}$ & 600 & accepted \\
13 & $23: 47$ & $\mathrm{R}$ & 600 & accepted \\
\hline
\end{tabular}

During the observations, the heliocentric distance of the comet was $R_{h}=1.87 \mathrm{au}$. The geocentric distance was $\Delta=1.71$ au and the phase angle was about $32^{\circ}$. The relatively small geocentric distance allowed to observe the faint comet (visible magnitude 15-16) with acceptable resolution. Indeed we could cover $1240 \mathrm{~km}$ on the comet image with one arcsec angular size, and one pixel representing about $370 \mathrm{~km}$ at the comet distance. The images obtained on the CCD were good enough to cover a field of 10.1 arcmin diameter corresponding to about $750000 \mathrm{~km}$ diameter at the comet distance.

In addition to the photometric features, we studied the colour index, $C$, of the comet in the red-near infrared domain, defined as the ratio of the scattered intensities:

$C=-2.5 \log \frac{S(R)}{S(I)}$,

in which the quantities $S(R)$ and $S(I)$ are the two recorded (calibrated) intensities of the light scattered by cometary dust particles through the two wavebands of $\mathrm{R}$ and I filters. Alternatively, the cometary colour index can be expressed by the relation:

$C=m_{R}-m_{I}$,

where the $m_{R}$ and $m_{I}$ are the cometary magnitudes observed through the $\mathrm{R}$ and I cometary filters.
Table 4. Observed magnitudes (obs. mag.) after airmass corrections and catalogue magnitudes (cat. mag.) for two photometric standard stars, along with the corrections $(\Delta m)$ to be applied to the photometric data analysis. 'av. $\Delta m$ ' denotes the average value of $\Delta m$ for the two stars.

\begin{tabular}{lccccc}
\hline Filters & star & obs. mag. & cat. mag. & $\Delta m$ & av. $\Delta m$ \\
\hline R & SA93424 & 11.747 & 11.066 & 0.681 & 0.6805 \\
R & SA96235 & 11.261 & 10.581 & 0.680 & \\
I & SA93424 & 11.468 & 10.562 & 0.906 & 0.9100 \\
I & SA96235 & 10.985 & 10.071 & 0.914 & \\
\hline
\end{tabular}

The light received from the comet is solar light scattered by the dust which has its own colour in the different wavelengths range (solar colours). To allow comparison between different observers and better determine specifically the dust properties, it is usual to consider the solar colour as a reference for zero intrinsic 'reddening' of the object. The solar colour has to be estimated in the wavelength range of our filters and subtracted from the colour index C. If the colour index is higher than the solar colour, we are speaking of reddening and $C_{n}$ (the normalised colour index) is positive. Holmberg (2006) using Sun-like stars measured the colour index of the Sun through different sets of filters. In particular the bandpass of the Sloan Digital Sky Survey (SDSS) filters are: red $((\lambda=625 \mathrm{~nm}, \Delta \lambda=140 \mathrm{~nm})$ and infrared ( $\lambda=770 \mathrm{~nm}, \Delta \lambda=150 \mathrm{~nm}$ ), close to the Bessell filters we used (Fukugita et al., 1996). For these SDSS filters, the solar magnitude difference is $C_{\text {Sun }}=0.12 \pm 0.01$. Our filters having slightly different bandpasses, we still use this value to correct $\mathrm{C}$ but increase the reported error bars to 0.02 .We define the normalised colour or reddening by $C_{n}=\mathrm{C}-C_{S u n}$.

\section{RESULTS AND DISCUSSIONS}

We previously reported photometric images of the comet $67 \mathrm{P}$ and gave light intensities expressed in arbitrary units using the same set of observations (Hadamcik et al. 2016). In the present work, we carry out absolute photometry in the two $\mathrm{R}$ and I bands in order to estimate the colour index, $C$, values for this comet.

Taking into account the uncertainties due the measurements techniques e.g. (i) the $\mathrm{R}$ and I filters do not have exactly the standard transmissions, (ii) A slight discrepancy exists between the colour of the standard G-type stars and the colour of the Sun, (iii) uncertainty on the solar colour value with the SDSS filters and also differences of transmission with our $\mathrm{R}$ and I filters. We estimate a total uncertainty smaller than $0.07 \mathrm{mag}$ and will take this value for each normalised colour $\left(C_{n}\right)$ value afterwards or to study the reddening of the dust. The corresponding uncertainty for the reflectivity gradient is of $5 \%$ per $100 \mathrm{~nm}$. In some cases we have to add the 'standard deviations' due to the photon noise.

\subsection{Photometric images}

Figure 2 reproduces photometric images of the comet through the two filters $\mathrm{R}$ and I, which are colour-coded to represent the absolute magnitudes per square arcsec of sky. 

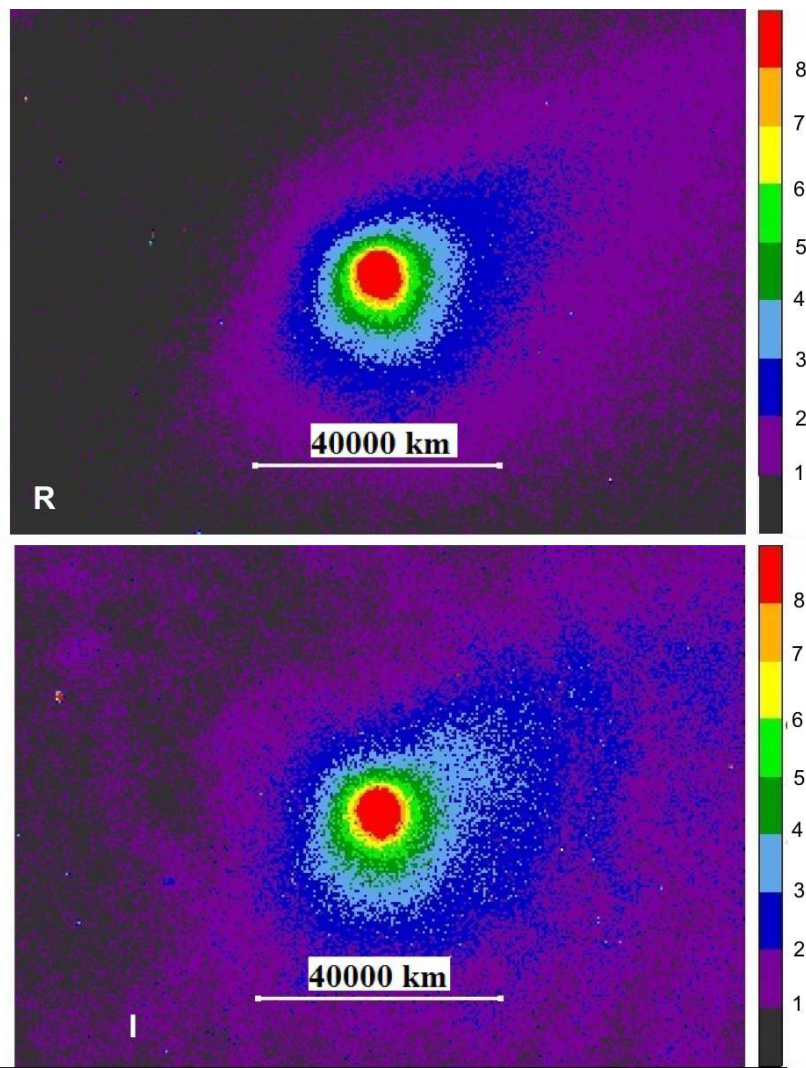

Figure 2. Intensity distribution of the comet $67 \mathrm{P}$ through $\mathrm{R}$ (upper figure) and I (bottom figure) filters. 100 pixels cover 37000 $\mathrm{km}$ at the comet. North is up, east is on the left and solar direction is south-east. The magnitude values are coded with colour scale from black to red representing small to large intensities per square arcsec. The faint fringes seen on the right side of the figure at the infrared wavelength prevented us to analyse the data farther than $20000 \mathrm{~km}$ from the optocentre.

The two images ( $\mathrm{R}$ and $\mathrm{I}$ ) exhibit similar structures with asymmetric coma and a well developed tail on a length more than $200000 \mathrm{~km}$ (Hadamcik et al. 2016 and Figure $3 \mathrm{a}$ ) at the position angle (PA) $300^{\circ} \pm 10^{\circ}$ (from north to east). The solar direction is $114.5^{\circ}$. On the the left corner of Figure 3, the main directions are indicated. The negative of the velocity vector is the direction of the projected orbit of the comet, its position angle is $301.6^{\circ}$. In consequence, the angle between the directions of the tail and the trail is about $1.6^{\circ}$. The trail particles are large and dark and were observed by Subaru's observatory (Moreno et al, 2017). They were previously ejected by the nucleus, but remained undisturbed to the solar radiation pressure and stayed along the cometary orbit which appears in projection as a straight line. The particles in the trail were ejected over many perihelion passages up to the present one, the particles closer to the nucleus trajectory being the younger ones. Within $20000 \mathrm{~km}$ distance of our study, the light scattered by the large dark trail particles is dominated by the light scattered by the smaller tail particles. The trail is not detected either at larger distances up to $200,000 \mathrm{~km}$ as on Figure 3a, only the tail is apparent.

In Figure 3b, isophotes are superimposed to the inten-
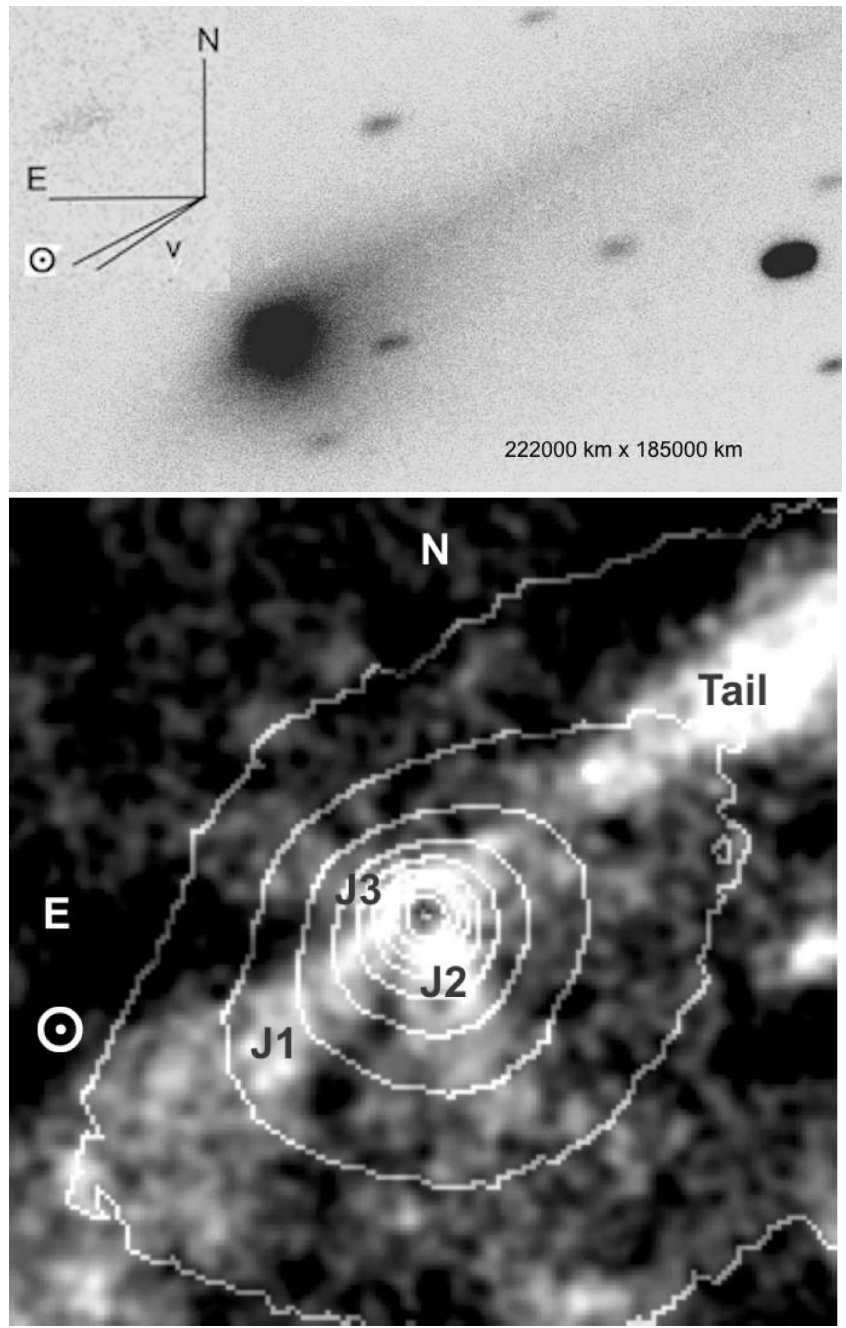

Figure 3. Top figure 3(a): the narrow long fine tail extends at more than $200000 \mathrm{~km}$ from the optocentre. In the left corner, orientations on the sky is shown and $\mathrm{v}$ is the velocity vector. Bottom figure 3(b): Isophototes superimposed on the treated intensity image by the rotational gradient method in the red $(\mathrm{R})$ wavelength. Field of view is 165 pixels and corresponds to $61000 \mathrm{~km}$. The directions of the main jets $(\mathrm{J} 1, \mathrm{~J} 2, \mathrm{~J} 3)$, tail and sunward directions (with mark $\odot$ ) are indicated.

sity image treated by the rotational gradient method (Larson \& Sekanina 1984) to emphasize the structures. Three jets are clearly detected. Their position angles are measured on the treated intensity images and confirmed on the isophotes:

- the main jet $(\mathrm{J} 1)$ direction is $135^{\circ} \pm 20^{\circ}$ (that is close to the solar direction)

- the direction of the second jet (J2) is $190^{\circ} \pm 20^{\circ}$

- a shorter jet $(\mathrm{J} 3)$ is found in the direction $70^{\circ} \pm 15^{\circ}$

On the isophotes graph, we can clearly detect the beginning of the tail. We performed the studies mainly in the inner part of the coma (less than $20000 \mathrm{~km}$ distance from the centre) because faint fringes are found at optocentric distances larger than $+20000 \mathrm{~km}$ on the I filter image (cf Fig. 2, bottom). 


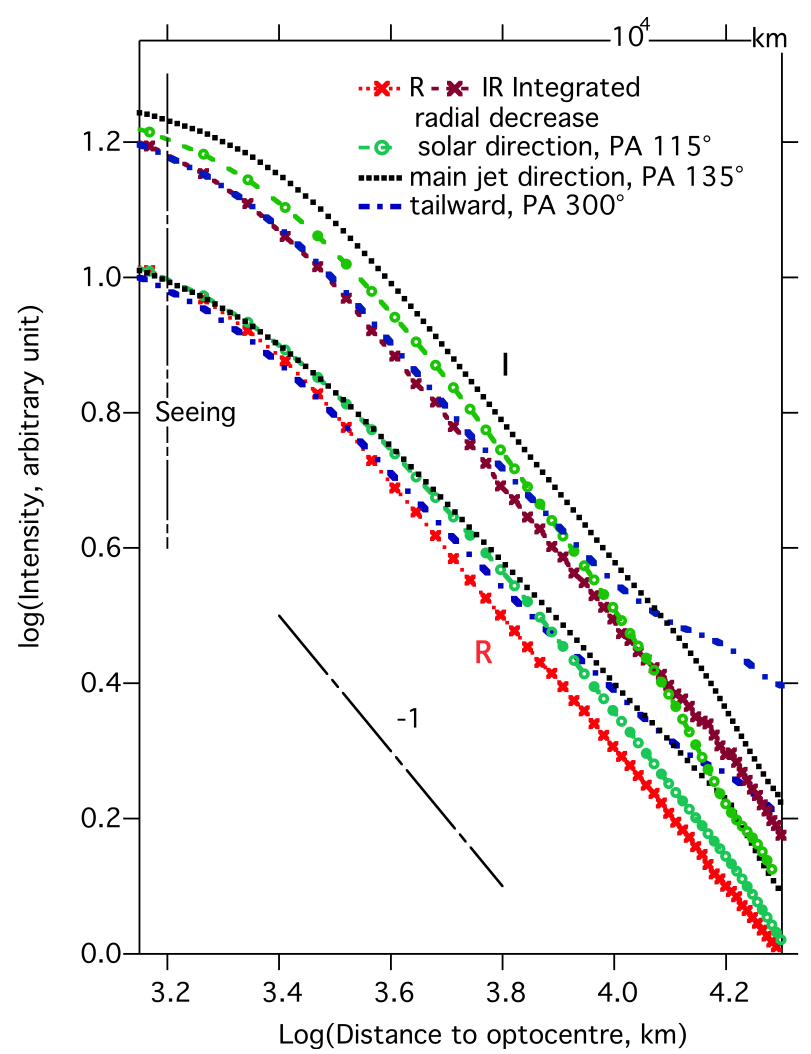

Figure 4. Radial profiles of the comet $67 \mathrm{P}$ through the $\mathrm{R}$ and I filters using a double-logarithmic scale (distances are in $\mathrm{km}$ ), The azimuthally-integrated intensities are measured using 1-pixel width annulus. The other profiles are measured along the structures (solar, tail and jet-1 directions).

\subsection{Radial profiles of intensity}

The R and I profiles (azimuthally-integrated profile, solar direction, tail direction and J1) are presented in Figure 4. They are obtained from the calibrated $\mathrm{R}$ and I intensity images. Slope values for azimuthally-integrated profile and tailward at different distances from the optocentre are given in Fig. 5. The distance interval used in the measurement of each profile is $368 \mathrm{~km}$, and the intensities have been integrated over an aperture of radius $1850 \mathrm{~km}$ such that random fluctuations are effectively smoothed out. The slope values are calculated in each position on the curve in a running mode. General trends are shown in Table 5, they are similar in both wavelengths. In particular, the slopes are comparable in values except for a few differences we shall discuss later. The azimuthally-integrated radial profiles decrease with a slope $\simeq-1$ indicating a quasi steady-state dust coma for both the filters in the range $3000-20000 \mathrm{~km}$ from optocentre. Theoretically, in a steady-state coma, the production of dust and its expansion would be constant, isotropic and homogeneous. In the case of $67 \mathrm{P}$, it means that the dust scattering properties are stable on average, without drastic changes during the ejection or expansion in the tail direction.

For distances from optocentre smaller than $3000 \mathrm{~km}$,
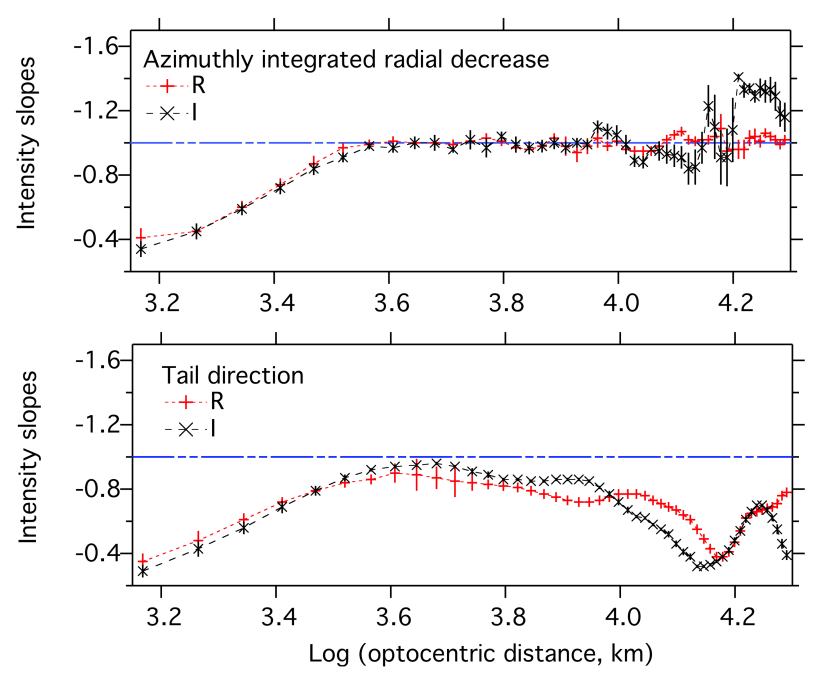

Figure 5. Slopes of various radial profiles observed with $\mathrm{R}$ and I filters. The data on this figure are the local slopes of the profile intensities as shown on Fig. 4. From top to bottom: azimuthallyintegrated and along the tail direction. For large distances from optocentre, the slope can exhibit spurious oscillations which result from weak fringes with the infrared filter (observation artefact) appearing in the edge of the field.

the slopes are all small due partially to atmospheric blurring. On (Fig 5 and Table 5), we can notice that the usual slope -1 is recovered very precisely on the azimuthally-averaged intensity, but this is not the case in the tail direction. This might result from dust directly ejected from the nucleus and/or pushed by solar radiation pressure from the coma and making the tail. On the tail profiles, a bump is clearly apparent at optocentric distances higher than $10000 \mathrm{~km}$ indicating a progressive increase of the cross-section of the dust (e.g. new particles pushed back by the solar radiation pressure, particles from a jet pushed in the tail direction... ). In the solar and jet-1 directions (Fig 4), the slopes increase gently for the small distances, then increase sharply around $14000 \mathrm{~km}$. This might be due to the shape of the jet-1, which appears to be curved from both the jet-1 initial direction and the solar direction (Hadamcik et al. 2016). The slope would then increase because of the sudden decreasing number density of dust particles.

\subsection{Colour index measurements}

In the sections below, we shall use the definition (1) or (2) of the colour index and calculate the normalised colour index $\left(C_{n}\right)$.

However, alternative definitions to estimate the colour of the dust or 'reddening' can be used as well: for example the 'normalised colour index' of the dust may be defined as 'the spectral slope', or 'normalised spectral reflectivity' (e.g. Jewitt \& Meech 1986 ; Ivanova et al. 2017a) which is the rate of change of the reflectivity gradient with respect to the wavelength interval (expressed in \% per $100 \mathrm{~nm}$ ). Precisely, the reflectivity gradient, $d S / d \lambda$, is defined through the formula (A'Hearn et al. 1984): 
Table 5. Slopes of different profiles observed with $\mathrm{R}$ and I filters.

\begin{tabular}{lccccr}
\hline $\begin{array}{l}\text { Radial } \\
\text { profile }\end{array}$ & filter & $\begin{array}{c}1800- \\
3000 \mathrm{~km}\end{array}$ & $\begin{array}{c}3000- \\
10000 \mathrm{~km}\end{array}$ & $\begin{array}{c}10000- \\
14000 \mathrm{~km}\end{array}$ & $\begin{array}{r}14000- \\
20000 \mathrm{~km}\end{array}$ \\
\hline $\begin{array}{l}\text { azimuth. } \\
\text { integrated }\end{array}$ & $\mathrm{I}$ & $-0.68 \pm 0.03$ & $-0.99 \pm 0.01$ & $-0.92 \pm 0.05$ & $-1.10 \pm 0.04$ \\
\hline sunward & $\mathrm{R}$ & $-0.68 \pm 0.04$ & $-0.99 \pm 0.01$ & $-1.01 \pm 0.01$ & $-1.00 \pm 0.02$ \\
& $\mathrm{I}$ & $-0.59 \pm 0.04$ & $-1.07 \pm 0.01$ & $-1.40 \pm 0.03$ & $-1.25 \pm 0.04$ \\
\hline jet 1 & $\mathrm{I}$ & $-0.59 \pm 0.03$ & $-0.88 \pm 0.01$ & $-1.08 \pm 0.01$ & $-1.19 \pm 0.01$ \\
\hline tailward & $\mathrm{R}$ & $-0.61 \pm 0.03$ & $-1.01 \pm 0.01$ & $-1.00 \pm 0.02$ & $-1.40 \pm 0.02$ \\
& $\mathrm{I}$ & $-0.64 \pm 0.04$ & $-0.89 \pm 0.01$ & $-0.89 \pm 0.01$ & $-1.31 \pm 0.03$ \\
\hline & $\mathrm{R}$ & $-0.65 \pm 0.03$ & $-0.81 \pm 0.01$ & $-0.67 \pm 0.01$ & $-0.57 \pm 0.02$ \\
& & & & & \\
\hline
\end{tabular}

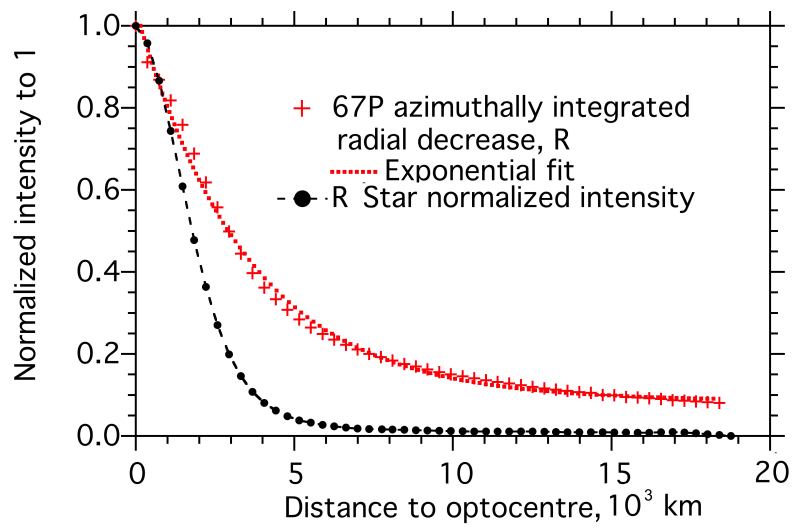

Figure 6. Azimuthally-integrated radial profile of the comet 67P through the $\mathrm{R}$ filter, and the point spread function for a star. The full width at half-maximum is $3600 \pm 500 \mathrm{~km}$.

$\frac{d S}{d \lambda}=\frac{10}{S_{\mathrm{av}}} \frac{S\left(\lambda_{2}\right)-S\left(\lambda_{1}\right)}{\lambda_{2}-\lambda_{1}}$

in which $S$ is the reflectivity, $S_{\text {av }}$ the average reflectivity over the wavelength interval $\left(\lambda_{1}, \lambda_{2}\right)$ expressed in micrometers. The coefficient 10 is added to normalise the reflectivity gradient parameter to a wavelength interval $100 \mathrm{~nm}$.

\subsubsection{Colour index determination method}

The average colour index through an aperture is calculated from the intensities given in two $\mathrm{R}$ and I calibrated images. For example, the normalised colour index of the comet for an aperture $22000 \mathrm{~km}$ diameter is $C_{n}=0.40 \pm 0.07$ (equivalently, the reflectivity gradient is found to be: $21.5 \% \pm 6 \%$ per $100 \mathrm{~nm})$.

To interpret the small values of the slopes close to the optocentre - corresponding to the colour indices at distances smaller than $3000 \mathrm{~km}$ we estimated the point-spread function using two bright stars present on the normalised frames. On the images, the star trails are elongated rectangles with half-disks at the two ends. We measured the point-spread function by averaging the values of the intensity in the rectangular region of the trail, in the direction perpendicular to it. On the Figure 6, the star profile is compared to the azimuthally-integrated profile of the comet.
Near the optocentre and up to $1200 \mathrm{~km}$ from it, the comet profile is close to the star profile. The light scattered from this central region can plausibly be attributed to the embedded comet nucleus. Further away, light scattered by the coma dust particles is responsible of the enlargement of the profiles as compared to the stars. Precisely, the full width at half maximum is measured to be $3600 \pm 500 \mathrm{~km}$ for $\mathrm{R}$ and $3900 \pm 500 \mathrm{~km}$ for I images. This is in good agreement with the seeing disk diameter measured by the Observatory at the same time (about $3300 \pm 350 \mathrm{~km}$ ). One can conclude that the solar light scattered by cometary dust is dominant for optocentric distances larger than $3000 \mathrm{~km}$ (with average colour index $0.38 \mathrm{mag}$ in a $6000 \mathrm{~km}$ diametre aperture). While contribution of the comet nucleus might be possible for distances less than $1200 \mathrm{~km}$, leading to the colour index $0.41 \mathrm{mag}$ in a $2400 \mathrm{~km}$ diametre aperture. According to Bertini et al. (2017), the comet nucleus should be redder than the coma dust. Then, for this comet, the impact of the nucleus on the coma colour seems to be negligible, even at small distances from the optocentre. Atmospheric turbulence may also partly explain the low value of the colour index obtained.

\subsubsection{Comet colour index map}

The two-dimensional colour index map of the comet $67 \mathrm{P}$ is presented in Fig. 7. To compare with the photometric features, isophotes and directions of the main structures are superimposed. Moreover, a white semi-transparent disc hides partially the inner region blurred by the seeing. Precise centering at the level of 0.1 pixel for both images through $\mathrm{R}$ and I filters, is mandatory to avoid artefacts. According to the data shown on the Fig. 7, the colour index ranges between $0.2 \mathrm{mag}$ to $0.7 \mathrm{mag}$, exhibiting an asymmetric coma between the solar and tail directions. These values represent the normalised colour index of the dust through the coma. The particular patterns observed in the colour map of the comet and discussed here, are reliable within an error bar of 0.07 mag (please refer beginning of Section 3).

To provide an easy way to show the variations of the colour index in the coma, we averaged the luminous flux through the two $\mathrm{R}$ and I filters over 3-pixels radius circular masks to calculate values in specific coma positions. This was done along the tail direction, along the jet- 1 direction and also in the North direction to illustrate the decrease 
of the colour index just outside the seeing disk region. The corresponding normalised colour index variations are shown on Fig. 8. In particular, a slight decrease of the colour index in the tail direction is clearly visible before a more regular increase. On the other hand, the colour index along the jet-1 direction is quite high close to the optocentre, then decreases strongly in agreement with the radial decrease in the jet-1 direction (Fig. 4).

We mentioned above that the azimuthally-integrated radial profiles exhibit slopes close to -1 between 3000 and $20000 \mathrm{~km}$. The difference between the R- and I-profiles is then almost constant in that distance range. It can be converted to normalised colour index, $C_{n}$ values: the colour index value is always in the range $0.35 \pm 0.07$ mag to $0.42 \pm 0.08$ mag up to about $10000 \mathrm{~km}$ from the optocentre. The value through a $22000 \mathrm{~km}$ diameter corresponds to $0.40 \pm 0.07$ mag.

On the other hand, the colour index values of the dust are not the same in different structures, e.g. comparing tail with jet-1. The colour index values also vary along each structure as a function of the optocentric distance.

This representation, using both the normalised colour index map and the isophotes, suggests unexpected effects which deserve discussion here:

- first, we note that the shape of the domain in blue coded colour on the Fig. 7. (that is the small values of the colour index) looks to follow the intensity isophotes superimposed on the colour map. This suggests that this small value of the colour index represents here the particles of the dust tail which are the most sensitive to the solar radiation pressure.

- the various structures seem to originate from the optocentre. This suggests that the different colour indices result from different properties of the particles evolving during the movement from their ejection place. Such typical different colour indices are for example the $0.53-0.66 \mathrm{mag}$ (the yellow-green coded colour index on the Fig. 7) in the jet- 1 direction, or the $0.20-0.44$ mag (the black-blue coded colour index on the Fig. 7) in the tail direction.

- in solar and jet-1 directions in Fig. 8, the colour index increases up to $2500 \mathrm{~km}$ from the optocentre, then decreases when the distance increases further (up to $14000 \mathrm{~km}$ ) and decreases sharply up to about $16000 \mathrm{~km}$ ) with the sharp increase of the infrared intensity slope in the profile. This is in contrast to the tail direction along which the colour index is about $0.28 \mathrm{mag}$ at a distance $1600 \mathrm{~km}$, then it decreases to about $0.25 \mathrm{mag}$ at $3000 \mathrm{~km}$ before increasing regularly even beyond $10000 \mathrm{~km}$ in regions where the slopes of intensities decrease. However, it is worth noting that the colour index of the dust is essentially 'red' in all the cases (that is with positive colour index).

- along the main structures (jet side and tail side), the colour index varies significantly when the dust is flowing away from the nucleus. For the jets, this is probably correlated to the changing characteristics of the particles freshly ejected from the nucleus or the size distribution of the particles in the different structures.

More precisely, the decrease of the colour index takes place up to $1000 \mathrm{~km}$ from the nucleus, then the change of colour index occurs in the region located between the site of ejec-

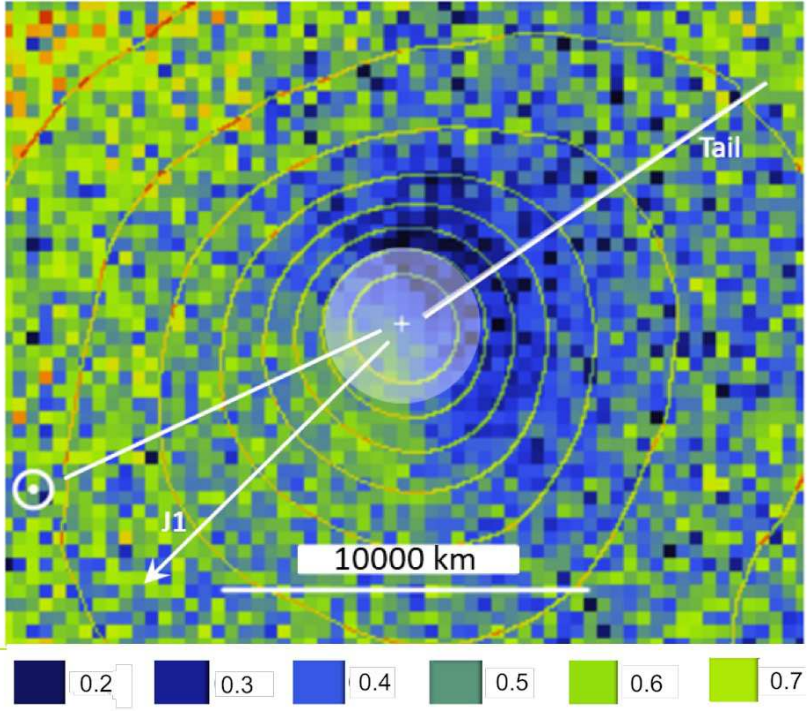

Figure 7. Normalised colour index map with isophotes contours superimposed. North is up, east on the left and solar direction (with the mark $\odot$ ) is south-east. The different colour indices are coded, the scale is shown below the image. The position of the nucleus (optocentre) is marked with the symbol + . The directions of the tail and of the main jet-1 (J1) are indicated on the figure.

tion and $1000 \mathrm{~km}$ after it. A first explanation for this behaviour may be the sublimation of ices contained in the dust particles, as was observed in some other comets. Nevertheless, the dust in $67 \mathrm{P}$ is very poor in ices, which was found mainly in pebbles close to the nucleus (Fulle et al. 2016b) and its sublimation was observed at less than $10 \mathrm{~km}$ in a jet (Gicquel et al. 2016). At more than $1000 \mathrm{~km}$ from the nucleus, several hours after their ejection (dust ejection speed is between 1 and $10 \mathrm{~m} / \mathrm{s}$, (Rotundi et al. 2015)) it is very improbable that ices remain to be sublimated. Another suggestion is the fragmentation of large fluffy aggregates leading to a number of new cometary particles which are both finer than the ejected parent particles and eventually with more absorbant refractive index values.

About the asymmetric coma seen in the colour index map, the north side exhibits much smaller colour indices than the South side. This feature might provide information about inhomogeneity of the particle distribution close to the nucleus, in the solar and anti-solar directions: lighter and/or some particular carbonaceous particles might be dominant in the North side, due to some reason (e.g. gravitation or interaction with the solar radiation pressure). There is here an interesting observation which deserves complementary work for definitive interpretation.

\subsection{Discussion}

\subsubsection{Evolution of the 67P post-perihelion morphology}

The overall shape of the coma is found similar to that observed one month before from HST observations (Hadamcik 


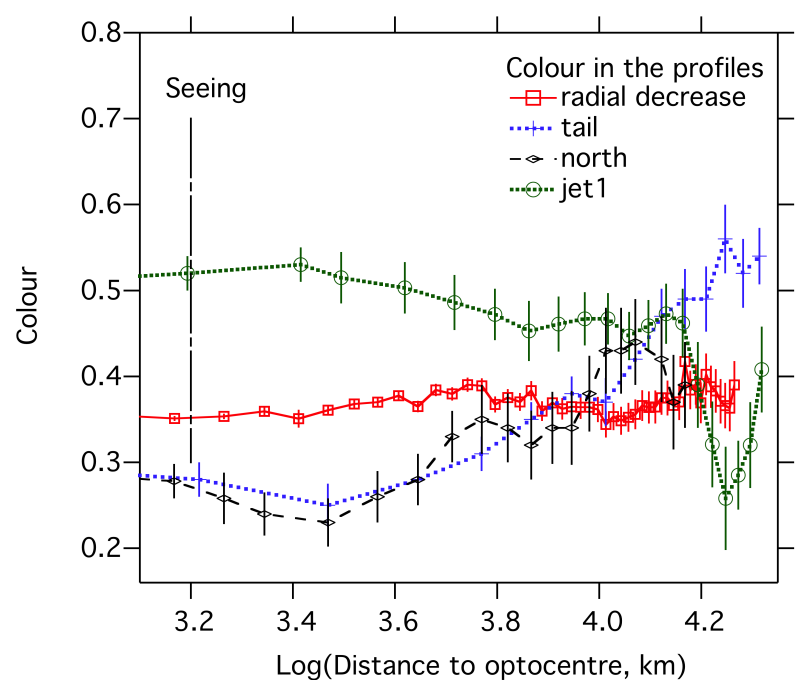

Figure 8. Various colour index profiles using the data on two normalised images to avoid artefacts. The error bars on the profiles represent the standard deviations

et al. 2016). In particular, the main jets are close to the same position angles. However, some jets appear to be less extended because their projection on the skychanged with the phase angle and their projected direction changed with the aspect angle of the nucleus. These structures have also been observed with about the same position angles during the Rosetta mission by several observers (e.g. Snodgrass et al. 2016). The two main curved jets are described for 20152016 apparition by Knight et al. (2017), Boehnhardt et al. (2016) and Rosenbush et al. (2017). The main jet remained close to the solar direction, curved and extended on the sky though it was progressively fading. Such features were also observed in previous apparitions, e.g. Schleicher (2006) observed a sunward radial feature in 1996 when the comet was close to perihelion, he also observed at the same time a sun-tail assymetry. Clearly visible features were observed by Agarwal (2007) in 2003, they interpreted them as border lines of coma fans from active areas of different latitude. During 2009-2010 apparition (Hadamcik et al 2010) several jet features were observed with some evolution from night to night.

\subsubsection{Colour index and dust properties in the different coma regions of $67 P$}

It was shown theoretically and through numerical simulations by Sen et al. (2017), that a decrease in the colour index is indicative of the increasing presence of finer dust particles while such decrease is not related to change in the particle porosity. Considering a typical size-distribution of the dust particles in the form of an inverse power-law (that is: $\sim 1 / a^{s}$ with the particle radius $a$ ), a decrease in the colour index may then be the sign of a larger exponent $s$. From another point of view, it seems then unlikely to use colourindex data to obtain precise information on the shape and structure (fluffy or compact) of the dust particles. These results are in agreement with the work of Kolokolova et al.
(1997) in which the authors concluded from their analysis that the cometary colour index is mainly determined by the size distribution of the cometary dust particles, whereas the cometary polarization variation is more complex involving particle size and shape.

It is of the utmost importance to have observables related to a limited number of parameters, then the colour index is a very interesting candidate. However, one has to be aware of the problem of the particle composition. Indeed, different colour index values can also result from different chemical compositions of the dust particles. In the domain of the wavelength range that we studied, reflectivity for various materials generally changes when the light wavelength increases from red to infrared. This is particularly true for the refractive indices of organic compounds which often change significantly in the near infrared domain. Further studies in this area are warranted to have more definite conclusions.

Assuming the particle composition does not vary much along each structure of the comet, the strong relation between the colour index and the exponent of the sizedistribution leads to analyse our data according to special scenarios:

- let us take the evolution of the colour index along the jet-1 direction as a first example (see Fig. 8). The colour index decreases regularly up to $14000 \mathrm{~km}$, then the index decreases more sharply before reaching eventually the value of the colour index for the azimuthally-integrated radial profile. Translated in terms of the exponent of the particle-size distribution, a scenario consistent with the data is that the exponent increases smoothly - for example through the regular fragmentation of big particles originated from the nucleus. At $14000 \mathrm{~km}$, another process starts to occur. It might be the release of small particles made of different chemical compounds which were embedded in the primitive big particles. The population of particles around the nucleus at a large distance from it, could be constituted mainly of these finer particles, hence the same value of the colour index in the azimuthally-integrated radial profile at that distance.

- another example is the evolution of the colour index in the tail direction (see Fig. 8). In this case, the colour index starts from a very low value (then high value exponent of the size-distribution) up to $3500 \mathrm{~km}$ from optocentre. Then the index increases gradually further. The disappearance of the smallest particles from the interconnecting region between the coma and the tail may be due to their higher velocity after ejection when pushed by the solar radiation pressure in the tail direction and finally forming the straight long structure at large distances from the nucleus (Fig 3) or if charged they can be disrupted by electrostatic forces and/or could be pushed by the solar wind. In that case, striae would be observed in the dust tail, which is not the case (Boehnhardt et al 2016).

Let us close this section with a simple comment: it is worth noting here that, unlike big compact particles, small Rayleigh particles scatter systematically more intense light of small wavelengths than light of large wavelengths. It results in a rule of thumb: for a given particle composition, large negative value of $C$ may be the signature of a collection of small scatterers. Such small particle are not nec- 
essarily free but can themselves form fluffy (non-compact) aggregates as well.

On the other hand, more complex cases (e.g. dust particles made of both Rayleigh and non-Rayleigh parts) need numerical simulations to get the colour index behaviour.

A consequence of this remark is that the dust particles ejected from comet $67 \mathrm{P}$ have probably more complicated structures than fractal aggregates of very small grains as confirmed by the Rosetta dust particles images. Indeed, we did not observe any negative colour index around the comet.

\subsubsection{Colour index and dust properties in $67 \mathrm{P}$ compared to other remote and in-situ observations}

These colour index values are then helpful to complement light scattering data from cometary dust particles, since, there are many cometary molecules having their own emission bands within the visible region. However, we have to be careful about a point: even if the comet $67 \mathrm{P}$ is rich in dust, contribution of the molecular emission bands must be negligible compared to the light scattered by the dust particles for the analysis be simple. In the case of the filters R or I that we used, the light scattered by cometary dust can be considered as essentially uncontaminated from cometary molecular emissions.

Colour index of cometary dust was measured by different groups during the successive apparitions of the comet and especially during its last apparition, while in-situ measurements were taken with the Rosetta mission. Colour index was often calculated for the 'visible' domain B-R (blue-red) and G-R (green-red), and it is somewhat complicated to make a direct comparison with the R-I domain that we used.

During the 2003 apparition of the comet $67 \mathrm{P}$, for waveband $\mathrm{R}-\mathrm{V}$ rather a neutral colour index $(\sim 0.061)$ has been reported from the post-perihelion observations (Lara et al. 2005). For the same apparition and around the same time of observation, Lamy et al. (2006) obtained an average colour index of 0.52 , but they reported day to day variations.

It has been already discussed in the Section 3.3, that cometary colour index can also be expressed as the 'spectral slope' or 'normalised spectral reflectivity'. Rosenbush et al. (2017) mentioned that normalised colour index, measured as $\%$ per $100 \mathrm{~nm}$, is often referred to as the dust colour index or reddening and accordingly they have reported the reddening (or spectral slope) of the coma to be about $8.2 \% / 100 \mathrm{~nm}$ in G-R wavelength range at the 33.2 degree phase angle of the comet, for observation during 2015-16. They also reported a reddening of $12.5 \pm 2 \%$ per $100 \mathrm{~nm}$ in November 2015, in the B-I domain and concluded that the particles were large compared to the wavelength. In this context, Schleicher (2006) found an average reddening of $(30 \pm 42) \%$ per 100 $\mathrm{nm}$ for the apparitions of 1982-1996, and Lamy et al. (2006), observing with HST, obtained a gradient of $32 \pm 6 \%$ per 100 $\mathrm{nm}$ for the coma dust 205 days after perihelion during the 2003 apparition at a phase angle of $4.8^{\circ}$ in the spectral range 540-670 nm.

One has to be aware that the values given above from different authors are not normalised in the same way. This is then difficult to make a precise comparison. However, as a rough comparison, let us note here that, as indicated in Section 3.3.1, we find a value of $21.5 \pm 6 \%$ per $100 \mathrm{~nm}$ in a $22000 \mathrm{~km}$ diameter aperture in acceptable agreement with other observations.

Boehnhardt et al. (2016) observed the comet during four nights $(2015 / 09 / 10,2015 / 09 / 12,2016 / 01 / 30$ \& $2016 / 05 / 07)$. They found a colour index value of the dust between 0.22 to 0.25 mag over different apertures between diameters of $5000 \mathrm{~km}$ and $20000 \mathrm{~km}$, without noting any systematic variations with aperture or period of observations. Their $\mathrm{R}$ and I filters are not exactly those that we used and their corresponding solar colour index was 0.11 mag. We found a higher reddening of about 0.40 mag.

Rosenbush et al. (2017) tried to interpret the correlated variations of polarization and colour index by numerical simulations of the scattered light. The authors used a model of polydisperse spherical particles with a power-law size distribution with sizes between $0.1 \mu \mathrm{m}$ to $1 \mathrm{~mm}$. As the power-law exponent becomes larger (making the smaller particles more abundant), the colour index decreases except for very large particles for which the polarization increases and then finally again decreases. They had presented G-R colour index maps for 8 November 2015. At the centre of the coma, the value $C \simeq 0.8$ is found, then the index decreases to $0.7-0.6$ at $20000 \mathrm{~km}$ distance. They also presented polarization maps for the same date and for 9 December 2015, very similar to our observation data. A slight increase in polarization in the inner part of the coma is observed (from $3.9 \pm 0.3 \%$ in November, to $4.1 \pm 0.4 \%$ in December), indicating a possible progressive small change in the dust properties. As a consequence, a decrease in colour index was expected. We found $0.47 \pm 0.02 \mathrm{mag}$ at the optocentre in December 2015 (Fig. 8) and $C \simeq 0.4 \mathrm{mag}$ over a $22000 \mathrm{~km}$ aperture with the $\mathrm{R}$ and I filters. These values appear to be in agreement with the data from Rosenbush et al. (2017).

In-situ colour index of the dust was also measured e.g. with OSIRIS instrument. Bertini et al. (2017) studying the scattering phase function from $360 \mathrm{~nm}$ to $760 \mathrm{~nm}$ wavelengths measured reddening of $11.9 \pm 0.6 \%$ per $100 \mathrm{~nm}$ on 14 December 2015. Frattin et al. (2017) also measured the reddening for individual particles and found a reddening of $12.9 \pm 0.2 \%$ in B-R range, and a value of $7.31 \pm 2 \%$ in the R-I range (649-882 $\mathrm{nm}$ filters, that is different from our filters). These data confirm a decrease of reddening with the increase of wavelength range as seen by remote observations (viz. Boehnhardt et al. 2016).

After perihelion, as the power-law exponent increases with more abundant smaller particles (Fulle et al. 2016; Della Corte et al. 2016); the colour index of the dust decreases, except for very large particles close to the nucleus. Thus it appears that in the coma and the structures (jets and tail) the local size distribution of the dust particles varies depending on the region and the distance to the optocentre as well as eventually their composition. 


\subsubsection{Colour index of the dust particles in $67 \mathrm{P}$ compared to colour index in some other comets and their variations}

Measurements of colour indices from other comets, have been interpreted previously in various ways, and we present some examples as illustrative comparison hereafter.

Kolokolova et al. (1997) compared the colour index and light polarization for five different comets. However, the colour index and polarization values were partially contaminated by possible gas emissions. The authors did not observe significant change of the colour index when the phase angle varied. They noted that comet $21 \mathrm{P} /$ Giacobini-Zinner presented a redder colour index than the four other comets of the study, though all the five comets presented reddening. Using Mie-model particles with T-matrix computation and a multifactor analysis, the authors concluded that the colour index depends mainly on the size of the particles. However, the behaviour of light polarization is more complex and should include at least the particle shapes to be analysed.

Lara et al. (2003) observed the comet $21 \mathrm{P}$ and found very typical reddening values in the range $12-19 \%$ per 100 $\mathrm{nm}$, except at some dates when dust appeared suddenly either bluer or redder than usual, without clear correlation to any sudden special activity of the nucleus.

Ivanova et al. (2017b) observed the comet C/2013 UQ4 (Catalina) and found a red colour index of the dust changing to a blue colour index after a couple of days. The authors attributed these unusual observations to a change in composition of the inhomogeneous nucleus. Blue colour index was attributed to $\mathrm{Mg}$-rich silicates and reddening to $\mathrm{MgFe}$-silicates and/or Kerogen and/or organics. The particle model was taken from Ivanova et al. (2016) with agglomerated debris and a size distribution power-law exponent of -2.4 . They also concluded that it is difficult to attribute a colour index to the dust with a single observation.

Lamy et al. (1987) studied the colour index in the tail of comet $1 \mathrm{P} /$ Halley. The authors observed reddening increasing with optocentric distance. Such a result was also obtained for the comet C/1975 V1 (West). Re-analysing the observations of Ney and Merril (1976), Zubko et al. (2014) concluded that the variations of the colour index and mainly a bluer colour of the dust, are due to solar radiation effect. The blue colour index was also observed for other comets.

Lara et al. (2011), observing comet 103P/Hartley 2, measured an average dust reddening of $24 \%$ per $100 \mathrm{~nm}$. Considering the colour index map and coma profiles, the bluer dust colour index was found to appear with increasing optocentric distance in the jet direction, while the colour index was almost constant in the tailward direction. The jet pointing to the sun showed dust reddening variations close to the optocentre where dust is freshly expelled from the nucleus. These refractory particles get bluer further away with changes in their optical properties.

\section{CONCLUSIONS}

The absolute photometry study in R-I wave-bands of the coma and the tail transition region of comet $67 \mathrm{P}$ obtained on 12 December 2015 allowed us to suggest some dust prop- erties and local changes in the size distribution of the particles.

(i) In a single wavelength, the slopes of decrease of intensities suggest :

- a steady-state coma between 3000 and $20000 \mathrm{~km}$ from the optocentre

- some fragmentation of large particles when fresh dust is emitted in the jet structure

- anti-sunward, a multiple origin of the dust which was directly ejected from the nucleus but also pushed back from the sunward direction and jets by the solar radiation pressure towards the tail

(ii) By comparison of the two wave-bands

- A reddening of the dust is evident in the coma and its structures when comparing the radial decreases in intensities. The normalised colour-index in a $22000 \mathrm{~km}$ diameter aperture is found to be $0.40 \pm 0.07$ mag.

- The colour map shows an asymmetry with lower colour-indices in the tail direction in a range 0.20 to $0.44 \mathrm{mag}$, and higher in the solar direction in a range 0.53 to $0.66 \mathrm{mag}$ in the jet - over distances from optocentre between 3000 to $14000 \mathrm{~km}$.

(iii) Considering the Rosetta results to describe the dust particles properties (high refractory/ices ratio, presence of large fluffy aggregates with micron- to tens of micron-sized constituent components of mixed materials, power-law index of the size distribution higher than -4 after perihelion indicating the presence of small particles), we suggest that the different reddening through the coma are mainly the result of different local size distributions.

\section{ACKNOWLEDGEMENTS}

The author AKS acknowledges IUCAA and IIA, India for providing funds and telescope facilities for cometary observation.

\section{REFERENCES}

Agarwal J., MÃijller M., GrÃijn E.,2007, Space Science Revue 128 , isuue $1-4,79$

A'Hearn M.F., Schleicher D.G., Feldman P.D., Millis R.L., Thompson Don T., 1984, Astrophys J., 89, 579.

A'Hearn M. F., Millis R.L., Schleicher D.G., Osip D.J., Birch P.V., 1995, Icarus, $118,223-270$

Bentley M.S. et al., 2016, Nature, 537,7618, 73.

Bertini I., et al., 2017, MNRAS, 469, 404.

Boehnhardt H., Riffeser A., Kluge, Ries C., Schmidt M., Hopp U., 2016, MNRAS, 462, 376.

Churyumov K., 2005, Kinematika i Fizika Nebesnykh Tel, Suppl 5,487 .

Della Corte V. et al., 2016, MNRAS 462, 210.

Fray N. et al., 2016, Nature 538, 7623,72.

Frattin E., et al., 2017, MNRAS, 469, 195.

Fukugita M., Ichikawa T., Gunn J.E., Doi M., Shimasaku K,. Schneider D.P., 1996, A J, 111, 1748.

Fulle M. et al., 2016a, ApJ, 821, 19 (14pp).

Fulle M. et al., 2016b, MNRAS, 462, S132.

Gicquel A. et al., 2016, MNRAS, 462, S57. 
Hadamcik E., Sen A.K., Levasseur-Regourd A.C., Gupta R., Lasue J., 2010, A\&A, 517, A86, 1-9.

Hadamcik E., Levasseur-Regourd A.C., Hines D.C., Sen A.K., Lasue J., 2016, MNRAS, 462, 507.

Hilchenbach M., et al., 2016, ApJ, 816, L32.

Holmberg J., Flynn C., and Portinari L., 2006, MNRAS, 367, 449.

Ivanova O.V. et al., 2016, PSS 121, 10.

Ivanova O.V., Rosenbush V.R., Kiselev N.N., Afanasiev V.L., Korsun P.P., 2017a, MNRAS, 469, 386.

Ivanova O.V., et al., 2017b, MNRAS, 469, 2695.

Jewitt D. and Meech K. J., 1986, ApJ 310, 937.

Knight M.M., Snodgrass C., Vincent J.B., Conn B.C., Skiff B.A., Schleicher D.G., Lister T., 2017, MNRAS, 469, 661.

Kolokolova L., Jockers K., Chernova G., and Kiselev N., 1997, Icarus $126,351$.

Lamy P.L, Gruen E., Perrin J. M., 1987, A\&A, 187, 767L.

Lamy P.L, Toth I., Weaver H.A., Jorda L., Kaasalainen M., Gutierrez P.J., 2006, A\&A, 458, 669.

Lara L.M., Licandro, J., Tozzi, G. P., 2003, A\&A, 404, 373L.

Lara L.M., Gutierrez P.J., de Leon J., Licandro J., 2005, EMP, 97, 165.

Lara L.M., Lin Z.Y., Meech K., 2011, A\&A, 532, 871.

Larson S.M and Sekanina Z., 1984, The Astronomical Journal 89, 571

Levasseur-Regourd, A. C., et al.,2018, Space Science Reviews, $214(3), 64$.

Mannel et al., 2016, MNRAS 462, S304.

Maquet L, 2015, A\&A, 579, A78.

Mor Moreno F. et al., MNRAS 469, S186, 2017

Mottola S. et al., 2014, A\&A, 569, L2 1-5.

Ney E.P. and Merrill K.M., 1976, Science, 194, 1051.

Osip D.J, Schleicher D. G and Millis R.L, 1992, Icarus, 98, 1, 115

Parihar, P. S., Sahu, D. K., Bhatt, B. C., Subramaniam, A., Anupama, G. C., Prabhu, T. P, 2003, BAS of India, 31, 453.

Rosenbush V.K., Ivanova O.V., Kiselev N.N., Kolokolova L.O., Afanasiev V.L., 2017, MNRAS, 469, 475.

Rotundi A. et al., 2015, Science, 347 (6220), aaa3905.

Schleicher D.G, 2006, Icarus, 181, 2, 442.

Schulz et al., 2015, Nature, 518, 216.

Sierks H. et al., 2015, Science, 347(6220), aaa1044

Sen A.K., Botet R., Vilaplana R., Choudhury N.R., Gupta, R, 2017, JQSRT, 198, 164.

Snodgrass C. et al, 2016, MNRAS 462, s138.

Snodgrass C. et al., 2017, Philosophical transactions of the Royal Society A, 375, 2097, 20160249.

Taylor M.G.G.T., Altobelli N., Buratti B.J., Choukroun M., 2017, Philosophical transactions of the Royal society A 375, 2097, 20160262.

Zubko E., Muinonen K., Videen G., Kiselev N.N., 2014, MNRAS, 440, 2928. 